\title{
Salicylic acid-independent plant defence pathways
}

\author{
Corné M.J. Pieterse and Leendert C. van Loon
}

\begin{abstract}
Salicylic acid is an important signalling molecule involved in both locally and systemically induced disease resistance responses. Recent advances in our understanding of plant defence signalling have revealed that plants employ a network of signal transduction pathways, some of which are independent of salicylic acid. Evidence is emerging that jasmonic acid and ethylene play key roles in these salicylic acid-independent pathways. Cross-talk between the salicylic acid-dependent and the salicylic acid-independent pathways provides great regulatory potential for activating multiple resistance mechanisms in varying combinations.
\end{abstract}

$\mathrm{P}$ lants possess multiple mechanisms to protect themselves against pathogen attack. Specific pathogen recognition mechanisms, governed by resistance gene products that interact with matching avirulence gene products from the pathogen, usually lead to a hypersensitive response at the site of pathogen invasion, keeping the pathogen isolated from the rest of the plant ${ }^{1}$. The formation of necrotic lesions, either as part of the hypersensitive response, or as a symptom of disease caused by a necrotizing virulent pathogen, is associated with the co-ordinated induction of an integrated set of defence responses: cell wall rigidification; synthesis of phytoalexins; and accumulation of pathogenesis-related proteins (PRs). Activation of defence-related genes extends systemically, conferring broad-spectrum resistance to viral, bacterial and fungal pathogens in distant, uninfected plant parts. Systemic acquired resistance $(\mathrm{SAR})^{2}$ is one of the best-studied signal transduction pathways involved in this complex resistance response, of which salicylic acid is a key signalling component ${ }^{3}$. Induction of SAR, by a predisposing infection with a necrotizing pathogen, coincides with an early increase in endogenously synthesized salicylic acid, not only at the site of primary infection but also systemically in the uninfected tissues ${ }^{4,5}$. Evidence for the key role of salicylic acid came from the analysis of transgenic plants expressing the bacterial $n a h G$ gene, which encodes the enzyme salicylate hydroxylase that inactivates salicylic acid by converting it to catechol. Transgenic NahG plants are unable to accumulate salicylic acid, and are also incapable of developing SAR, indicating that salicylic acid accumulation is required for the expression of $\mathrm{SAR}^{6}$.

NahG plants have also been valuable tools in the discovery of novel, salicylic acid-independent defence pathways that, like SAR, confer broad-spectrum systemic resistance. Recently, several research groups demonstrated that specific defence responses are unaffected by the absence of salicylic acid in the NahG plants, which indicates that these defence reactions operate independently of salicylic acid. This is supported by the discovery that several defence responses can be activated without an increase in the level of salicylic acid or salicylic acid marker gene expression. Plant growth regulators, jasmonic acid and ethylene, are now emerging as important signalling molecules in these alternative defence pathways.

\section{Salicylic acid-independent activation of $P R$ gene expression}

PRs accumulate in pathological or related situations and were identified originally as novel proteins that accumulated in tobacco leaves reacting hypersensitive to tobacco mosaic virus (TMV) ${ }^{7}$. Some of these PRs, such as chitinases and glucanases, possess antimicrobial activity, and are thought to contribute to the broad-spectrum resistance that is triggered upon infection with a necrotizing pathogen.
Pathogen-induced expression of most $P R$ genes is correlated with the onset of SAR in uninfected tissue, and can be mimicked by exogenous application of salicylic acid ${ }^{8}$. Nonetheless, several lines of evidence suggest that salicylic acid is not the only signal involved in the induction of $P R$ genes. Firstly, $P R$ gene activation does not always coincide with enhanced levels of salicylic acid 9 Secondly, the set of $P R$ genes activated upon pathogen infection does not always match those activated by salicylic acid or its functional analogues, 2,6-dichloroisonicotinic acid (INA) and benzothiadiazole $(\mathrm{BTH})^{10,11}$. For instance, infection of tobacco by the soft-rot pathogen Erwinia carotovora or treatment of tobacco leaves with its elicitors, systemically induces a set of $P R$ genes that is different from that induced by exogenous application of salicylic acid ${ }^{12,13}$. During the first 12 hours after infection, Erwinia specifically activates a basic $\beta$-1,3-glucanase $(P R-2)$ and a basic chitinase $(P R-3)$ gene but not the salicylic acid-inducible $P R-1$ gene. Whereas, exogenous application of salicylic acid triggers the expression of $P R-1$ but not that of the $P R-2$ or the $P R-3$ gene. Interestingly, Erwinia-mediated induction of $P R$ genes is antagonized by salicylic acid, whereas salicylic acid-induced $P R$ gene expression is antagonized by Erwinia-derived elicitors ${ }^{12}$. The induction of Erwinia-mediated $P R$ gene expression is not affected in transgenic NahG tobacco plants. Moreover, Erwinia elicitors can enhance systemic resistance in wild type and transgenic NahG tobacco plants ${ }^{13}$, indicating that the signalling pathway is salicylic acid independent.

Another example in which $P R$ genes appear to be activated in a salicylic acid-independent manner has been demonstrated in transgenic tobacco plants expressing the pokeweed antiviral protein (PAP) from Phytolacca americana. PAP is a ribosome-inactivating protein that inhibits translation by catalytically depurinating eukaryotic ribosomes ${ }^{14}$. Transgenic plants expressing PAP are resistant to a broad spectrum of plant viruses and show enhanced resistance to the fungal pathogen Rhizoctonia solani, which is correlated with constitutive expression of $P R$ genes. Interestingly, PAP plants do not show elevated levels of salicylic acid, suggesting that $P R$ gene expression in these PAP transgenics is not dependent on salicylic acid ${ }^{15}$. Additional evidence for the observation that enzymatic activity of PAP is capable of inducing salicylic acid-independent defence responses came from grafting experiments. In these experiments, it was demonstrated that wildtype tobacco scions grafted onto PAP-expressing rootstocks show enhanced resistance to TMV and potato virus $\mathrm{X}$ infection in the absence of $P R$ gene expression and increased salicylic acid accumulation $^{16}$. However, it cannot be ruled out that PAP induces enhanced sensitivity to salicylic acid, leading to an increase in salicylic acid responses in the absence of elevated salicylic acid levels. 
In Arabidopsis and tobacco, $P R-1$ gene expression is particularly responsive to salicylic acid and is therefore often used as a marker for the salicylic acid-dependent SAR response $^{2} . P R-1$ gene expression in tobacco is controlled by at least two distinct signalling pathways. Polyacrylic acid (PAA) is a chemical inducer of $P R-1$ gene expression and enhances resistance to TMV in tobacco at the application site. One mechanism by which PAA might activate $P R-1$ gene expression is by inducing the accumulation of salicylic acid because PAAtreated plants also show elevated salicylic acid levels. However, in wild type and transgenic NahG tobacco plants, PAA is equally capable of inducing $P R-1$ protein accumulation ${ }^{17}$, suggesting the presence of more than one pathway leading to $P R-1$ gene expression, of which one is independent of salicylic acid.

Several lines of evidence indicate that the plant growth regulators jasmonic acid and ethylene play an important role. For instance, methyl jasmonate, a naturally occurring derivative of jasmonic acid, enhances the level of salicylic acid-induced $P R-1$ gene expression in tobacco ${ }^{18}$. Moreover, combinations of methyl jasmonate and ethylene synergistically induce members of both the $P R-1$ and the $P R-5$ gene family in this species. Recently, it was demonstrated that the salicylic acid-independent activation of $P R$ genes by Erwinia elicitors involves a concerted action of jasmonic acid and ethylene (E.T. Palva, pers. commun.), strongly supporting earlier evidence ${ }^{19}$.

\section{Jasmonic acid- and ethylene-regulated plant defensins and thionins}

Over the past few years, evidence has accumulated demonstrating that jasmonic acid and ethylene are indeed important signals in the induction of systemic defence responses. Both are rapidly produced when the plant is attacked by a pathogen, particularly during necrotizing infections where the rise in jasmonic acid levels even extends to systemic tissues ${ }^{20}$. Moreover, exogenous application of these signalling molecules induces a set of defence genes that are also activated upon pathogen infection, among which are genes encoding plant defensins and thionins: small, cysteine-rich, basic proteins with antimicrobial activity ${ }^{21,22}$. In Arabidopsis, the thionin gene Thi2.1 and the plant defensin gene PDF1.2 are locally and systemically activated after infection with a necrotizing pathogen or exogenous application of methyl jasmonate, but not after the leaves have been treated with salicylic acid ${ }^{20,23,24}$ (Fig. 1). In Arabidopsis NahG plants, pathogen-induced systemic activation of PDF1.2 is unaffected ${ }^{20}$, indicating that this

\section{Panel I}

(a)

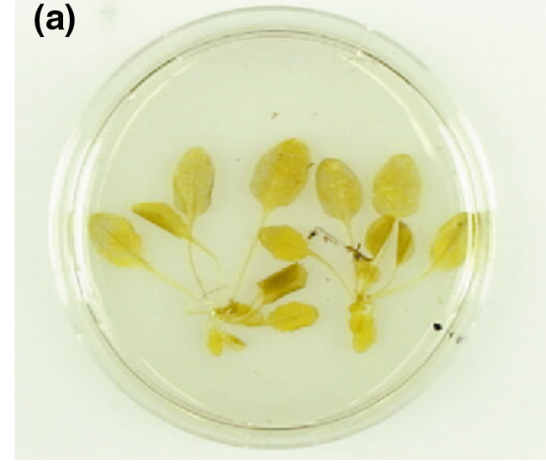

(b)

(c)

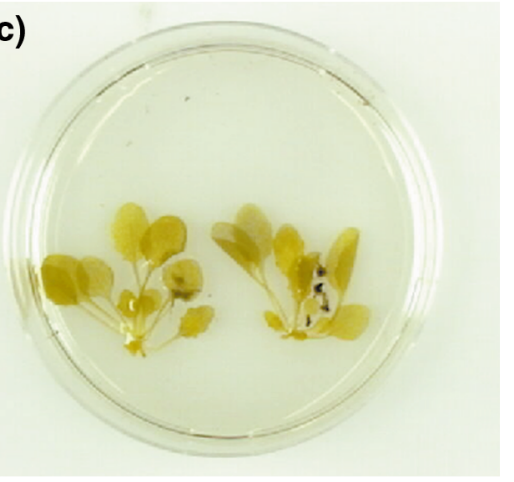

(d)

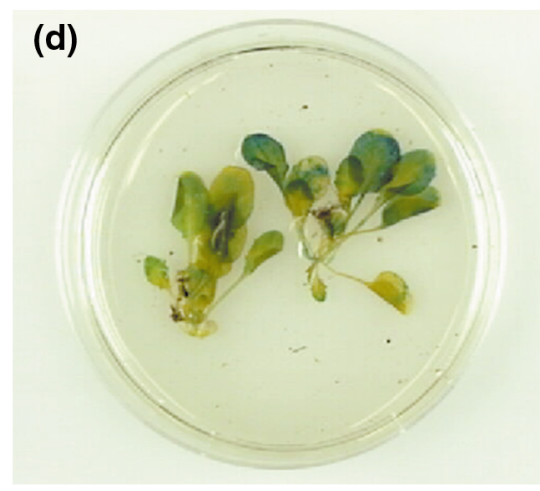

\section{Panel II}

(a)
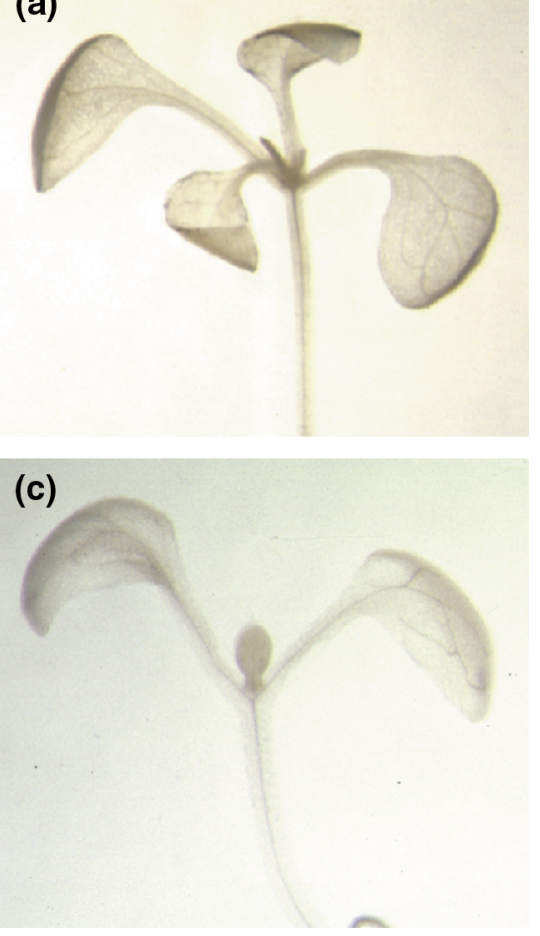

(d)
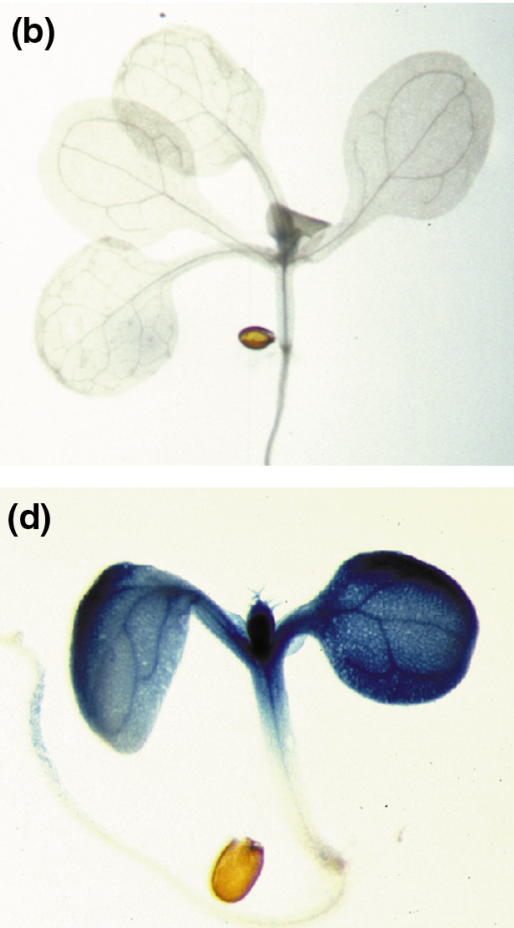

Fig. 1. Characterization of GUS reporter-gene expression in transgenic Arabidopsis lines harbouring a PDF1.2 (Panel I) or Thi2.1 (Panel II) promoter-GUS gene construct. Plants were treated with: (a) water; (b) $1 \mathrm{mM}$ salicylic acid; (c) $0.1 \%$ ethanol; (d) $50 \mu \mathrm{M}$ methyl jasmonate in $0.1 \%$ ethanol. GUS reporter-gene activation is visualized by the accumulation of a blue colour. Clearly, both the PDF1.2 and the Thi2.1 promoter are activated by methyl jasmonate but not by salicylic acid, suggesting that $P D F 1.2$ and Thi2.1 gene expression are both controlled by a salicylic acid-independent signalling pathway. Photographs courtesy of W.F. Broekaert and I.A.M.A. Penninckx (PDF1.2-GUS), and H. Bohlmann, A. Vignutelli and M. Nibbe (Thi2.1-GUS). 


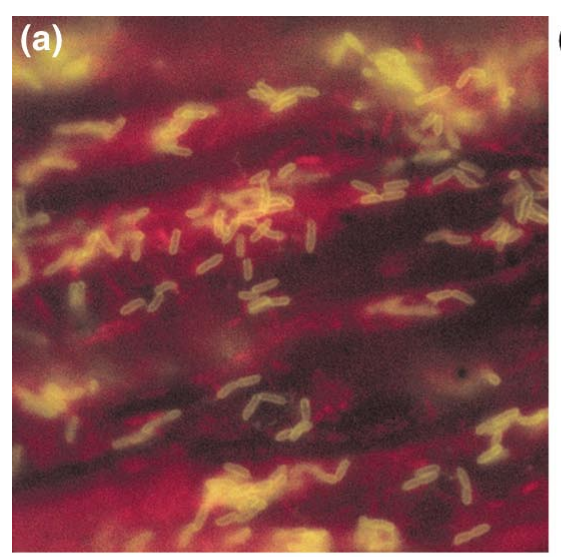

(b)

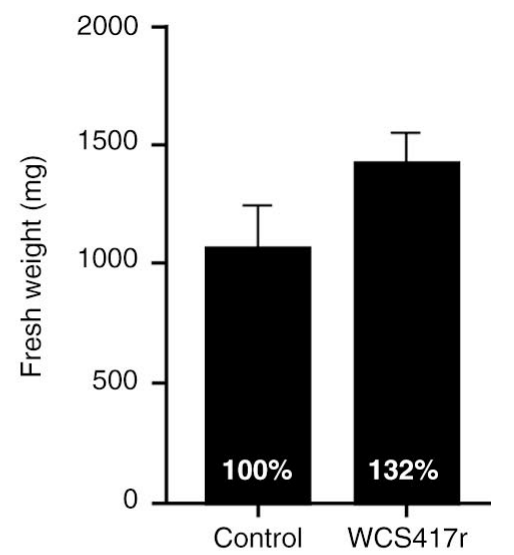

(c)

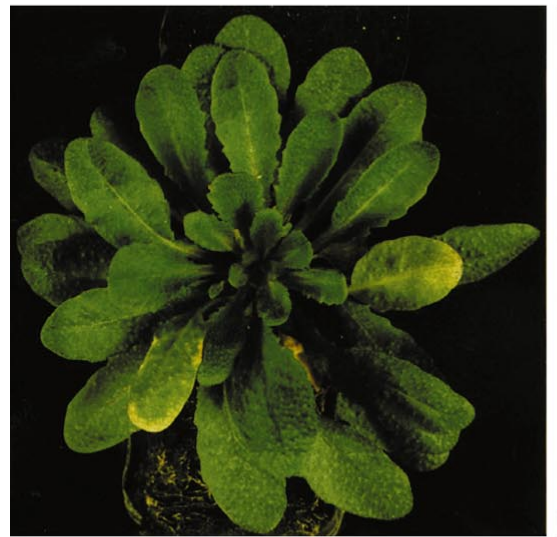

(d)
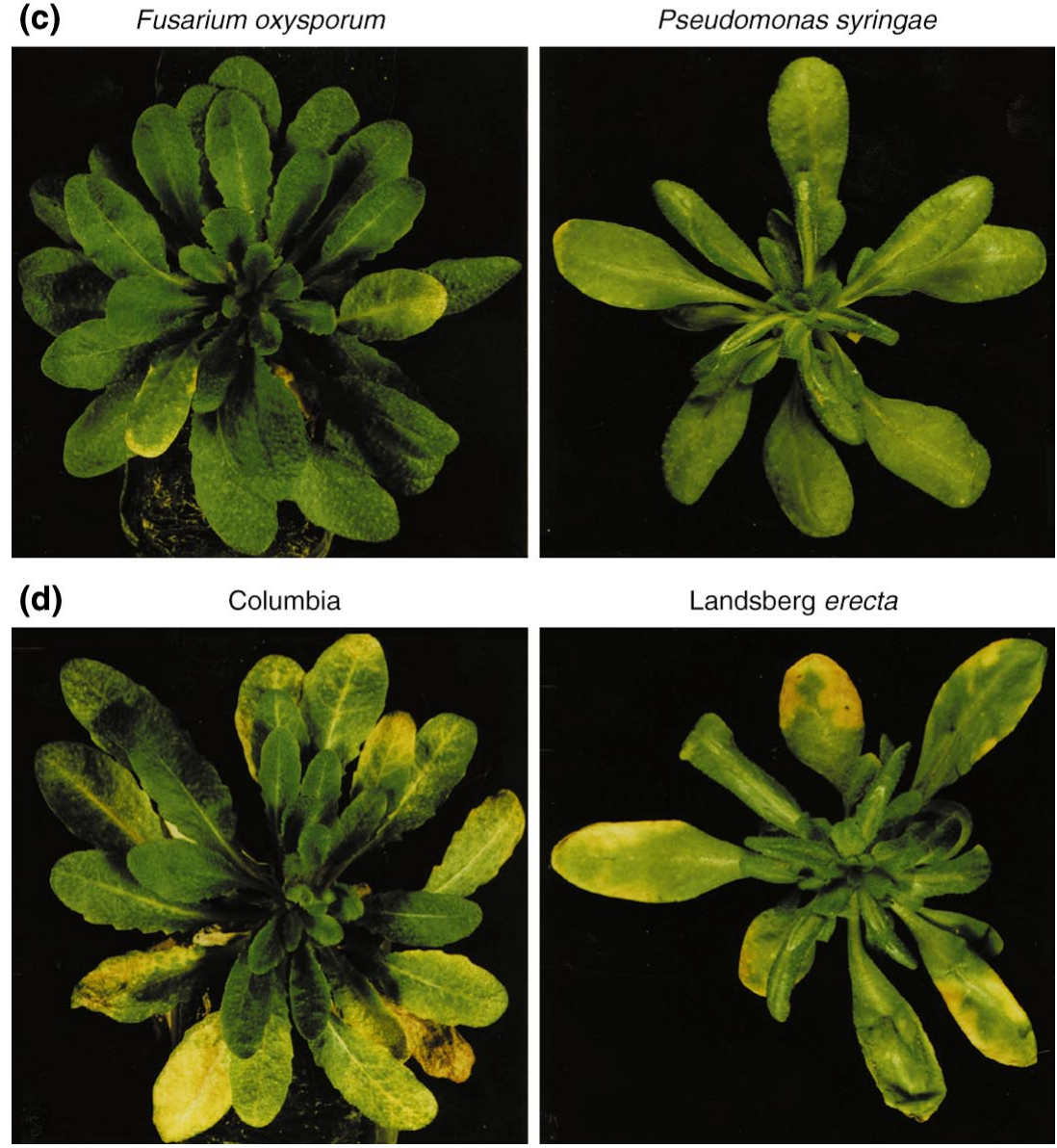

Fig. 2. (a) Pseudomonas fluorescens WCS417r bacteria on the surface of a plant root. Rhizobacteria are visualized by the green fluorescence of FITC-labelled antibodies that were directed against the target bacterium. (b) Plant growth-promoting effect on Arabidopsis plants grown in soil containing $P$. fluorescens WCS417r bacteria. (c) P. fluorescens WCS417rmediated induced systemic resistance (ISR) in Arabidopsis ecotypes Columbia and Landsberg erecta against infection by the fungal root pathogen Fusarium oxysporum f.sp. raphani and the bacterial leaf pathogen Pseudomonas syringae pv. tomato. Treatment of the roots with $P$. fluorescens WCS417r results in the elicitation of ISR, visualized by a strong reduction of the symptoms caused by the challenging pathogens compared with the control (d). Parts (c) and $(d)$ are reproduced, with permission, from Ref. 46. response. Recently, it was demonstrated that the jasmonate and ethylene signalling pathways need to be triggered concomitantly to activate $P D F 1.2$ upon pathogen infection ${ }^{25}$.

Infection with a necrotizing pathogen triggers both salicylic acid-dependent $P R$ gene expression and salicylic acid-independent PDF1.2 and Thi2.1 gene expression. This raises the question as to whether the respective pathways share certain signalling steps. If this is the case, which signalling components do they share and where do these pathways diverge? Experiments designed to dissect genetically the signalling pathways controlling systemically induced disease resistance, have greatly contributed to our understanding. Mutant screens, which were aimed at identifying potential signalling components of the SAR pathway in Arabidopsis, yielded both loss-of-function mutants and gain-of-function mutants ${ }^{26}$. Loss-of-function mutants, such as the allelic mutants $n p r l$ and niml, are unable to mount systemic resistance or express $P R$ genes after pathogen infection or salicylic acid treatment, indicating that they are blocked in the SAR signalling pathway. Gain-of-function mutants, such as those from the cpr and cim series, constitutively express $P R$ genes and are more resistant to pathogen infection, indicating that these mutants constitutively express SAR. Experiments using $n p r l$ and cprl mutants demonstrated that pathogen-induced systemic activation of $P D F 1.2$ is unaltered, indicating that these mutations in the SAR pathway do not affect PDF1.2 gene expression ${ }^{20}$. The signalling pathways leading to $P R$ and $P D F 1.2$ gene expression are distinct. However, Arabidopsis SAR mutants cpr5, cpr6 and $a c d 2$ from the gain-of-function mutant class show constitutive expression of $P R$ genes as well as PDF1.2 (Refs 20,27, 28) and Thi2.1 (Ref. 28), suggesting that the corresponding signalling pathways are connected, probably in the early steps of their signal transduction pathways. Pathogeninduced PDF1.2 gene expression is tightly correlated with the rise in endogenous jasmonic acid levels ${ }^{20}$, whereas $P R$ gene expression is strictly correlated with elevated levels of salicylic acid ${ }^{27}$. Mutants cpr5 and acd 2 form spontaneous lesions that give rise to increased levels of both salicylic acid and jasmonic acid ${ }^{20,27}$, indicating that the early signal transduction step that both pathways have in common is probably the initiation of lesion formation. regulatory pathway is salicylic acid independent. Interestingly, $P D F 1.2$ gene expression is blocked in the ethylene-insensitive mutant ein 2 and the jasmonic acid-insensitive mutant coil (Ref. 20), demonstrating that the signalling pathway involved in PDF1.2 induction requires components of the ethylene and jasmonic acid

\section{Systemic defence responses induced by wounding}

Defence responses are triggered after wounding, some of which are expressed systemically. A classic example is the observation that following herbivore attack, tomato leaves systemically accumulate proteinase inhibitor (pin) proteins that reduce further 
insect feeding ${ }^{29}$. Wound-induced systemic resistance against fungal pathogens has recently been demonstrated as well. Wounding of young rice plants induces a systemic resistance response that leads to protection against infection by the rice blast fungus Magnaporthe grisea in the absence of $P R$ gene expression ${ }^{30}$. Jasmonic acid has emerged as an important signal in the wound response ${ }^{31}$. Wounding not only causes a rapid production of jasmonic acid but the levels of ethylene increase as well. Recently, it was demonstrated that neither wounding, nor jasmonic acid is able to induce pin gene expression in the presence of inhibitors of ethylene action. By itself, ethylene is unable to activate pin gene expression, indicating that ethylene must sensitize the tissue to the inducing action of jasmonic acid ${ }^{32}$.

\section{Interactions between signals}

Both wounding and pathogen attack involve the production of jasmonic acid and ethylene. However, several lines of evidence indicate that their respective response pathways are distinct, or even antagonistic. For instance, in tobacco, wounding and pathogen attack show differential activation of different members of the $P R$ gene families ${ }^{33}$. Wounding appears to activate basic $P R$ genes, whereas pathogen attack predominantly leads to acidic $P R$ gene expression. A possible cause might be that upon wounding only jasmonic acid and ethylene appear to play a role, whereas after pathogen infection salicylic acid is produced as well. Salicylic acid and its functional analogues inhibit jasmonic acid-induced defence gene expression ${ }^{34-36}$. In Arabidopsis, the expression of the jasmonic acid- and ethylene-inducible gene PDF1.2 is enhanced in salicylic acid non-accumulating $\mathrm{NahG}$ plants ${ }^{20}$, suggesting that in wild-type plants pathogen-induced PDF1.2 gene expression is, to some degree, suppressed by endogenous salicylic acid. This is supported by the observation that PDF1.2 mRNA accumulation is inhibited upon application of the salicylic acid analogue INA (Ref. 27). However, synergistic effects of jasmonic acid and ethylene on salicylic acid-inducible responses have been reported as well. In tobacco, a combined treatment with methyl jasmonate and salicylic acid results in a stronger induction of $P R-1$ gene expression than treatment with salicylic acid alone ${ }^{18}$. Moreover, ethylene sensitizes Arabidopsis plants for salicylic acid action, leading to a higher level of $P R-1$ mRNA accumulation after salicylic acid treatment ${ }^{37}$.

\section{Systemic resistance induced by non-pathogenic rhizobacteria}

The capacity of plants to express a broad-spectrum, systemic resistance after primary infection with a necrotizing pathogen is well known. A relatively unknown form of induced disease resistance is triggered by non-pathogenic, root-colonizing rhizobacteria and is commonly referred to as rhizobacteria-mediated induced systemic resistance (ISR) ${ }^{38}$. Rhizosphere bacteria are present in large numbers on the root surface (Fig. 2a), where plant exudates and lysates provide nutrients. Certain strains of rhizosphere bacteria stimulate plant growth (Fig. 2b), and are therefore often referred to as plant growth-promoting rhizobacteria (PGPR). Selected rhizobacterial strains with biological control activity, mainly fluorescent Pseudomonas spp., reduce plant diseases by suppressing soil-borne pathogens through competition for nutrients, siderophore-mediated competition for iron or antibiosis ${ }^{39}$. Some of these strains are also able to reduce disease through a plant-mediated mechanism that is phenotypically similar to SAR, as the induced resistance extends to the above-ground plant parts and is effective against different types of plant pathogens ${ }^{38}$ (Fig. 2c). Some rhizobacteria trigger the SAR pathway by producing salicylic acid at the root surface ${ }^{40,41}$, and in other cases, ISR-inducing rhizobacteria trigger a different signalling pathway that does not require salicylic acid ${ }^{42-45}$.

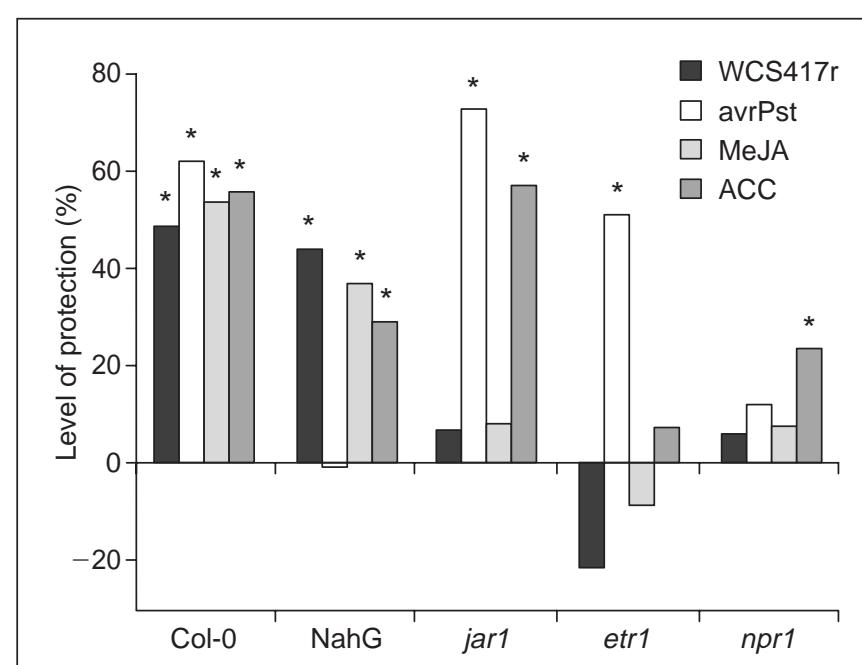

Fig. 3. Level of induced protection against infection by Pseudomonas syringae pv. tomato in different Arabidopsis genotypes. Wild-type Arabidopsis Col-0 plants, and genotypes altered in their response to either salicylic acid (NahG), jasmonic acid (jarl), ethylene (etrl) or inducers of systemic acquired resistance (SAR; $n p r l)$ were treated with non-pathogenic, induced systemic resistance (ISR)-inducing rhizobacteria by growing them in soil containing $P$. fluorescens WCS417r bacteria. SAR was induced three days before challenge inoculation by pressure infiltrating three lower leaves per plant with the avirulent pathogen $P$. syringae pv. tomato (avrPst) carrying the avirulence gene avrRpt2. Chemical treatments were performed three days before challenge inoculation by dipping the leaves of fiveweek-old plants in a solution containing either $100 \mu \mathrm{M}$ methyl jasmonate (MeJA) or $1 \mathrm{mM}$ of the ethylene precursor 1-aminocyclopropane-1-carboxylate (ACC). Challenge inoculations were performed by dipping the leaves of five-week-old plants in a bacterial suspension of the virulent pathogen $P$. syringae pv. tomato. Four days after challenge, plants were evaluated on the severity of disease symptoms. The level of protection is given as a reduction of disease symptoms compared to control-treated plants. In contrast to pathogen-induced SAR, rhizobacteria-mediated ISR follows a salicylic acid-independent, and jasmonic acid-dependent and ethylenedependent signalling pathway. Like SAR, rhizobacteria-mediated ISR is regulated by NPR1. Methyl jasmonate-induced protection is blocked in jarl, etrl and nprl plants, whereas ACC-induced protection is affected in etrl and $n p r l$ plants, but not in jarl plants. This illustrates that components from the jasmonic acid response and the ethylene response act in sequence and upstream of NPR1 in activating resistance against $P$. syringae pv. tomato.

*Statistically significant differences in disease severity compared with the non-treated control plants (Fisher's LSD test, $\alpha=0.05$ ).

The existence of a salicylic acid-independent ISR pathway induced by non-pathogenic rhizobacteria has been clearly demonstrated in Arabidopsis. In contrast to pathogen-induced SAR, ISR triggered by Pseudomonas fluorescens strain WCS417r or $P$. putida strain WCS358r is not associated with the activation of $P R$ genes $^{42,43}$. Moreover, NahG plants that are unable to express SAR, develop normal levels of ISR after treatment of the roots with either of these ISR-inducing rhizobacterial strains ${ }^{42,43}$ (Fig. 3). Jasmonic acid and ethylene also appear to play an important role in this salicylic acid-independent pathway. The Arabidopsis jasmonate response mutant jarl and the ethylene response mutant etrl, which show a normal response to inducers of SAR, are unable to express ISR after root treatment with P. fluorescens WCS417r (Ref. 46), indicating that signal transduction leading to rhizobacteria-mediated ISR requires responsiveness to both jasmonate and ethylene. Although ISR and SAR seem to follow distinct signalling pathways, 




Fig. 4. Model showing systemic signalling pathways that can be induced in plants by non-pathogenic rhizobacteria, pathogen infection or wounding, such as caused by foraging insects. In all cases, a salicylic acid-independent pathway is triggered that involves both jasmonic acid (JA) and ethylene. Infection with a necrotizing pathogen predominantly results in the activation of the salicylic acid (SA)-dependent systemic acquired resistance response (SAR) leading to the accumulation of salicylic acid-inducible PRs. In addition, jasmonic acid- and ethylene-inducible defence responses are triggered that might be similar to those induced by non-necrotizing pathogens. Depending on the invading pathogen, the composition of defensive compounds produced after pathogen infection could vary between predominantly salicylic acid-inducible and jasmonic acid- or ethylene-inducible (green bar at the bottom). In this respect, jasmonic acid and ethylene have a positive effect on the action of salicylic acid, whereas salicylic acid seems to have a negative effect on jasmonic acid- and ethylene-inducible defences. Wounding also results in the activation of jasmonic acid- and ethylene-inducible defence responses. However, the composition differs from that induced upon pathogen infection (blue bar at the bottom). This is probably because jasmonic acid and ethylene are the dominant signals during the wound response, while the levels of salicylic acid do not rise. In the rhizobacteria-mediated induced systemic resistance (ISR) pathway, components from the jasmonic acid and ethylene response act in sequence in activating a systemic resistance response that, like pathogen-induced SAR, is dependent on the regulatory protein NPR1. The ISR pathway shares signalling events with pathways that are initiated upon pathogen infection but is not associated with the activation of genes encoding plant defensins, thionins or PRs. This indicates that ISR-inducing rhizobacteria, such as Pseudomonas fluorescens WCS417r, trigger a novel signalling pathway leading to the production of so far unidentified defensive compounds (bottom yellow box). Unlike pathogen attack and wounding, this ISR response seems to be associated with an increase in sensitivity to jasmonic acid and/or ethylene rather than an increase in their production, which might lead to the activation of a different set of defence genes. Whether some of these unknown defensive compounds are also produced upon pathogen attack or wounding needs to be investigated. Signalling pathways 1-5 adapted from: (1) Refs 42,43,46; (2) Refs 2,20,23,26; (3) Refs 12,13; (4) Refs 24,29,31,32; (5) Refs 18,34-37.

they are both blocked in the SAR regulatory mutant $n \operatorname{prl}$ (Ref. 46; Fig. 3). Thus, the regulatory factor NPR1 (also called NIM1) ${ }^{47}$ is not only required for the SA-dependent expression of $P R$ genes that are activated during SAR, but also for the jasmonate- and ethylenedependent activation of so far unidentified defence responses resulting from rhizobacteria-mediated ISR.

In Arabidopsis, the sequence of signalling events in the ISR pathway leading to resistance against the bacterial leaf pathogen Pseudomonas syringae $\mathrm{pv}$. tomato has been investigated by application of methyl jasmonate and the ethylene precursor 1-aminocyclopropane1-carboxylate (ACC) as inducing agents ${ }^{46}$ (Fig. 3), which, like $P$. fluorescens $\mathrm{WCS} 417 \mathrm{r}$, are effective in inducing resistance against $P$. syringae pv. tomato in both wild-type and NahG plants, suggesting that they trigger the same salicylic acid-independent pathway. Analysis of the resistance-inducing capacity of methyl jasmonate and ACC in the mutants jarl, etrl and nprl, revealed that in this signalling pathway components from the jasmonic acid response and the ethylene response act sequentially and upstream of NPR1.
In spite of the fact that both the SAR and the ISR signalling pathways require the regulatory factor NPR1, the defence responses that are activated downstream of NPR1 are clearly different. $P R$ genes are activated in the SAR pathway but not in the ISR pathway, which suggests that the associated defence responses are differentially regulated by NPR1 depending on the signalling pathway that is activated upstream of NPR1. Sequence analysis revealed that NPR1 contains a functionally important ankyrin-repeat domain that might be involved in protein-protein interactions ${ }^{47,48}$. Binding of pathway-specific proteins to NPR1 might therefore account for the separation of the SAR and ISR pathways downstream of NPR1. It would be interesting to investigate what signalling components from the SAR and ISR pathways confer this specificity in NPR1-dependent defence gene activation.

Rhizobacteria-mediated ISR and pathogen-induced activation of the PDF1.2 gene are both salicylic acid-independent, but jasmonic acid- and ethylene-dependent defence reactions. Although they seem to share certain signalling steps, the corresponding signal transduction pathways are not identical. Rhizobacteria-mediated 
ISR requires NPR1 (Ref. 46), whereas PDF1.2 activation is an NPR1-independent defence response ${ }^{20}$. Most importantly, ISR is not associated with PDF1.2 gene expression ${ }^{46}$, indicating that the signalling pathways controlling ISR and PDF1.2 gene expression are at least partly distinct. This might be because, unlike pathogeninduced activation of PDF1.2, rhizobacteria-mediated ISR in Arabidopsis does not coincide with a strong increase in the levels of jasmonic acid and ethylene ${ }^{46}$. This suggests that ISR is associated with an increase in sensitivity to these hormones rather than an increase in their production, which might lead to the activation of a partially different set of defence genes.

\section{Outlook}

Recent advances in plant defence signalling pathway research have shown that plants are capable of differentially activating distinct defence pathways (Fig. 4). Depending on the type of invader encountered, the plant appears to be capable of switching on the appropriate pathway or combination of pathways. The plant signalling molecules salicylic acid, jasmonic acid and ethylene play an important role in this signalling network: blocking the response to either of these signals can render plants more susceptible to pathogens and even insects ${ }^{6,49-52}$. Resistance conferred by the salicylic acid-dependent pathway might be directed more against certain types of pathogens, whereas resistance conferred by salicylic acid-independent pathways might operate more effectively against other types of pathogens. Recently, evidence supporting this notion was obtained using Arabidopsis genotypes that are blocked in either the jasmonic acid or the salicylic acid response ${ }^{53}$. The jasmonic acid response mutant, coil, lost some of its basal resistance against the necrotrophic fungal pathogens Alternaria brassicicola and Botrytis cinerea, whereas basal resistance against the biotrophic fungus Peronospora parasitica was unchanged. In contrast, mutant $\mathrm{nprl}$ and transgenic NahG plants, that are both blocked in their response to salicylic acid, show a lower level of basal resistance against $P$. parasitica, whereas basal resistance against $B$. cinerea and $A$. brassicicola is unaffected. This indicates that the defensive compounds produced by the salicylic acid-dependent and -independent pathways have different specificities. A challenging question for the near future will be: how are plants adapted to switch on the right combination of defence pathways after encountering a certain pathogen? In this respect, the observed cross-talk between the signalling compounds salicylic acid, jasmonic acid and ethylene offers great regulatory potential. Therefore, research on the interplay between the pathways that are activated by these signalling molecules will provide important information.

\section{Acknowledgements}

We wish to thank Willem Broekaert, Holger Bohlmann and Tapio Palva for communicating unpublished information. We also thank Saskia van Wees, Jurriaan Ton, Hans van Pelt, Karen Léon-Kloosterziel, Boet Glandorf and Peter Bakker for useful discussions and helpful comments on this review.

\section{References}

1 De Wit, P.J.G.M. (1997) Pathogen avirulence and plant resistance: a key role for recognition, Trends Plant Sci. 2, 452-458

2 Ryals, J.A. et al. (1996) Systemic acquired resistance, Plant Cell 8, 1809-1819

3 Durner, J., Shah, J. and Klessig, D.F. (1997) Salicylic acid and disease resistance in plants, Trends Plant Sci. 2, 266-274

4 Malamy, J. et al. (1990) Salicylic acid: a likely endogenous signal in the resistance response of tobacco to viral infection, Science 250, 1002-1004

5 Métraux, J-P. et al. (1990) Increase in salicylic acid at the onset of systemic acquired resistance in cucumber, Science 250, 1004-1006

6 Gaffney, T. et al. (1994) Requirement of salicylic acid for the induction of systemic acquired resistance, Science 261, 754-756
7 van Loon, L.C. (1997) Induced resistance in plants and the role of pathogenesis-related proteins, Eur. J. Plant Pathol. 103, 753-765

8 Ward, E.R. et al. (1991) Coordinate gene activity in response to agents that induce systemic acquired resistance, Plant Cell 3, 1085-1094

9 Vallélian-Bindschedler, L., Métraux, J-P. and Schweizer, P. (1998) Salicylic acid accumulation in barley is pathogen specific but not required for defensegene activation, Mol. Plant-Microbe Interact. 11, 702-705

10 Schweizer, P., Buchala, A. and Métraux, J-P. (1997) Gene-expression patterns and levels of jasmonic acid in rice treated with the resistance inducer 2,6-dichloroisonicotinic acid, Plant Physiol. 115, 61-70

11 Schaffrath, U., Freydl, E. and Dudler, R. (1997) Evidence for different signaling pathways activated by inducers of acquired resistance in wheat, $\mathrm{Mol}$. Plant-Microbe Interact. 10, 779-783

12 Vidal, S. et al. (1997) Salicylic acid and the plant pathogen Erwinia carotovora induce defense genes via antagonistic pathways, Plant J. 11, 115-123

13 Vidal, S. et al. (1998) Cell wall-degrading enzymes from Erwinia carotovora cooperate in the salicylic acid-independent induction of a plant defense response, Mol. Plant-Microbe Interact. 11, 23-32

14 Bonnes, M.S. et al. (1994) Pokeweed antiviral protein inactivates pokeweed ribosomes; implications for the antiviral mechanism, Plant J. 5, 173-183

15 Zoubenko, O. et al. (1997) Plant resistance to fungal infection induced by nontoxic pokeweed antiviral protein mutants, Nat. Biotechnol. 15, 992-996

16 Smirnov, S., Shulaev, V. and Tumer, N.E. (1997) Expression of pokeweed antiviral protein in transgenic plants induces virus resistance in grafted wildtype plants independently of salicylic acid accumulation and pathogenesisrelated protein synthesis, Plant Physiol. 114, 1113-1121

17 Malamy, J. et al. (1996) Dissection of the salicylic acid signaling pathway in tobacco, Mol. Plant-Microbe Interact. 9, 474-482

$18 \mathrm{Xu}$, Y. et al. (1994) Plant defense genes are synergistically induced by ethylene and methyl jasmonate, Plant Cell 6, 1077-1085

19 Brederode, F.T., Linthorst, H.J.M. and Bol, J.F. (1991) Differential induction of acquired resistance and PR gene expression in tobacco by virus infection, ethephon treatment, UV light and wounding, Plant Mol. Biol. 17, 1117-1125

20 Penninckx, I.A.M.A. et al. (1996) Pathogen-induced systemic activation of a plant defensin gene in Arabidopsis follows a salicylic acid-independent pathway, Plant Cell 8, 2309-2323

21 Terras, F.R.G. et al. (1995) Small cysteine-rich antifungal proteins from radish: their role in host defense, Plant Cell 7, 573-588

22 Epple, P., Apel, K. and Bohlmann, H. (1997) Overexpression of an endogenous thionin enhances resistance of Arabidopsis against Fusarium oxysporum, Plant Cell 9, 509-520

23 Epple, P., Apel, K. and Bohlmann, H. (1995) An Arabidopsis thaliana thionin gene is inducible via a signal transduction pathway different from that for pathogenesis-related proteins, Plant Physiol. 109, 813-820

24 Vignutelli, A. et al. (1998) Systemic and local induction of an Arabidopsis thionin gene by wounding and pathogens, Plant J. 14, 285-295

25 Penninckx, I.A.M.A. et al. (1998) Concomitant activation of jasmonate and ethylene response pathways is required for induction of a plant defensin gene in Arabidopsis, Plant Cell 10, 2103-2114

26 Dong, X. (1998) SA, JA, ethylene, and disease resistance in plants, Curr. Opin. Plant Biol. 1, 316-323

27 Bowling, S.A. et al. (1997) The cpr5 mutant of Arabidopsis expresses both NPR1-dependent and NPR1-independent resistance, Plant Cell 9, 1573-1584

28 Clarke, J.D. et al. (1998) Uncoupling PR gene expression from NPR1 and bacterial resistance: characterization of the dominant Arabidopsis cpr6-1 mutant, Plant Cell 10, 557-569

29 Ryan, C.A. (1992) The search for the proteinase-inhibitor inducing factor, PIIF, Plant Mol. Biol. 19, 123-133

30 Schweizer, P. et al. (1998) Induced systemic resistance in wounded rice plants, Plant J. 14, 475-481

31 Wasternack, C. and Parthier, B. (1997) Jasmonate-signalled plant gene expression, Trends Plant Sci. 2, 302-307

32 O'Donnell, P.J. et al. (1996) Ethylene as a signal mediating the wound response of tomato plants, Science 274, 1914-1917 
33 Bol, J.F. et al. (1996) Regulation of the expression of plant defense genes, Plant Growth Regul. 18, 87-91

34 Peña-Cortés, H. et al. (1993) Aspirin prevents wound-induced gene expression in tomato leaves by blocking jasmonic acid biosynthesis, Planta 191, 123-128

35 Doares, S.H. et al. (1995) Salicylic acid inhibits synthesis of proteinase inhibitors in tomato leaves induced by systemin and jasmonic acid, Plant Physiol. 108, 1741-1746

36 Niki, T. et al. (1998) Antagonistic effect of salicylic acid and jasmonic acid on the expression of pathogenesis-related (PR) protein genes in wounded mature tobacco leaves, Plant Cell Physiol. 39, 500-507

37 Lawton, K.A. et al. (1994) Acquired resistance signal transduction in Arabidopsis is ethylene independent, Plant Cell 6, 581-588

38 van Loon, L.C., Bakker, P.A.H.M. and Pieterse, C.M.J. (1998) Systemic resistance induced by rhizosphere bacteria, Annu. Rev. Phytopathol. 36, 453-483

39 Bakker, P.A.H.M., Van Peer, R. and Schippers, B. (1991) Suppression of soilborne plant pathogens by fluorescent Pseudomonads: mechanisms and prospects, in Biotic Interactions and Soil-Borne Diseases (Beemster, A.B.R. et al., eds), pp. 217-230, Elsevier

40 De Meyer, G. and Höfte, M. (1997) Salicylic acid produced by the rhizobacterium Pseudomonas aeruginosa 7NSK2 induces resistance to leaf infection by Botrytis cinerea on bean, Phytopathology 87, 588-593

41 Maurhofer, M. et al. (1998) Salicylic acid biosynthetic genes expressed in Pseudomonas fluorescens $\mathrm{P} 3$ improve the induction of systemic resistance in tobacco against tobacco necrosis virus, Phytopathology 88, 678-684

42 Pieterse, C.M.J. et al. (1996) Systemic resistance in Arabidopsis induced by biocontrol bacteria is independent of salicylic acid accumulation and pathogenesis-related gene expression, Plant Cell 8, 1225-1237

43 Van Wees, S.C.M. et al. (1997) Differential induction of systemic resistance in Arabidopsis by biocontrol bacteria, Mol. Plant-Microbe Interact. 10, 716-724

44 Hoffland, E. et al. (1995) Induced systemic resistance in radish is not associated with accumulation of pathogenesis-related proteins, Physiol. Mol. Plant Pathol. 46, 309-320
45 Press, C.M. et al. (1997) Salicylic acid produced by Serratia marcescens 90-166 is not the primary determinant of induced systemic resistance in cucumber or tobacco, Mol. Plant-Microbe Interact. 10, 761-768

46 Pieterse, C.M.J. et al. (1998) A novel signaling pathway controlling induced systemic resistance in Arabidopsis, Plant Cell 10, 1571-1580

47 Ryals, J.A. et al. (1997) The Arabidopsis NIM1 protein shows homology to the mammalian transcription factor inhibitor IкB, Plant Cell 9, 425-439

48 Cao, H. et al. (1997) The Arabidopsis NPR1 gene that controls systemic acquired resistance encodes a novel protein containing ankyrin repeats, Cell 88, 57-63

49 Knoester, M. et al. (1998) Ethylene-insensitive tobacco lacks nonhost resistance against soil-borne fungi, Proc. Natl. Acad. Sci. U. S. A. 95, 1933-1937

50 McConn, M. et al. (1997) Jasmonate is essential for insect defense in Arabidopsis, Proc. Natl. Acad. Sci. U. S. A. 94, 5473-5477

51 Staswick, P.E., Yuen, G.Y. and Lehman, C.C. (1998) Jasmonate signaling mutants of Arabidopsis are susceptible to the soil fungus Pythium irregulare, Plant J. 15, 747-745

52 Vijayan, P. et al. (1998) A role for jasmonate in pathogen defense of Arabidopsis, Proc. Natl. Acad. Sci. U. S. A. 95, 7209-7214

53 Thomma, B.P.H.J. et al. (1998) Separate jasmonate-dependent and salicylatedependent defense response pathways in Arabidopsis are essential for resistance to distinct microbial pathogens, Proc. Natl. Acad. Sci. U. S. A. 95, $15107-15111$

Corné M.J. Pieterse* and Leendert C. van Loon are at the Section of Plant Pathology, Dept of Plant Ecology and Evolutionary Biology, Utrecht University, PO Box 800.84, 3508 TB Utrecht, The Netherlands.

*Author for correspondence (tel +3130253 6887; fax +3130251 8366; e-mail c.m.j.pieterse@bio.uu.nl; internet http://www.bio.uu.nl/ fytopath).

\title{
Rhodopsin-mediated photosensing in green flagellated algae
}

\author{
Oleg A. Sineshchekov and Elena G. Govorunova
}

\begin{abstract}
Green flagellated algae possess a primitive visual system that regulates the activity of their motor apparatus. Photoexcitation of a rhodopsin-type photoreceptor protein gives rise to the photoreceptor current, which, above a certain threshold of stimulus intensity, induces the flagellar current. It is probable that the photoinduced alteration in flagellar beating is governed by changes in intracellular $\mathrm{Ca}^{2+}$ concentration. This rhodopsin-mediated sensory system serves to align the swimming path with the direction of the light stimulus, whereas processes of energy metabolism determine whether the oriented movement is directed towards or away from the light source.
\end{abstract}

$\mathrm{S}$ urvival of phototrophic organisms depends on their ability to optimize their exposure to sunlight. Light controlled cell motility appeared early in evolution as one of the strategies used to achieve this goal. Unicellular flagellated algae, as well as motile zoospores and gametes of macroalgae, actively search for optimal light conditions by means of phototaxis (i.e. oriented swimming towards or away from the light source $)^{1,2}$. In addition, a sudden change in light intensity, irrespective of its direction, usually elicits a photophobic or photoshock cell response, which appears as a transient stop, often followed by a brief period of backward motion ${ }^{1}$. Most flagellates rotate around their longitudinal axis during forward swimming. The ability to track the direction of light is based on the capacity to sense a temporal difference in the amount of quanta captured in a single photoreceptive region of the cell under lateral illumination. Structural and functional aspects of phototactic sensory systems found in different taxonomic groups of algae are very diverse ${ }^{2}$ and might reflect their independent evolutionary origin. Chlorophyceae are a unique group of 